I Consejo Nacional de Investigaciones Científicas y

Técnicas (CONICET), Universidad de Buenos Aires (UBA),

Instituto de Investigaciones Gino Germani (IIGG),

Buenos Aires, Argentina

spignuoli@conicet.gov.ar

https://orcid.org.0000-0002-9918-0931

\title{
SÍMBOLO Y COMUNICACIÓN - HACIA UN CONCEPTO DE COMUNICACIÓN SIMBÓLICA DESDE LA TEORÍA GENERAL DE SISTEMAS SOCIALES
}

\section{INTRODUCCIÓN}

En este trabajo examinamos los conceptos de símbolo y de simbolización de la Teoría General de Sistemas Sociales de Niklas Luhmann (en adelante: TGSS) con el propósito de conectarlo de manera sistemática con el concepto general de comunicación. Subrayamos, por tanto, desde un comienzo, que la TGSS cuenta con un concepto de símbolo. Esto podría sorprender a primera vista, pues la raigambre del concepto en la antropología filosófica o en visiones interpretativas, comprensivas o discursivas del mundo social podría llevar a la suposición de que es repelido por las posiciones sistémicas. Lo cierto es que Luhmann y Parsons, así como otros tantos destacados autores inscriptos en la sociología de sistemas, elaboraron sus propios conceptos de símbolo. En el caso de Luhmann, el símbolo qua concepto es alojado en las entrañas de la teoría de los Medios de Comunicación Simbólicamente Generalizados (en adelante: MCSG) y queda inscripto así en la teoría general de la sociedad. Esta ubicación privilegiada, orientada por cierto a un objeto tan vasto y complejo como la sociedad, no debería, sin embargo, hacernos perder de vista el hecho de que la sociedad es un objeto específico de la TGSS, y, más allá de la pretensión de generalidad de la teoría abocada a ella, toda definición dada en su seno es una definición específica, no una general. Esto concierne a todos los conceptos, incluido el de símbolo y el de simbolización (también irónicamente al de generalización). Es indispensable dar una definición general de ellos y, a tal efecto, debe situárselos al nivel de la teoría general de sistemas sociales. 
Esto no significa que Luhmann haya desatendido el concepto de símbolo; su aparición es recurrente en textos de distintas etapas de su carrera. Si bien tempranamente fue asociado con la generalización de sentido, en materiales anteriores a los años I980 no se aprecia en el término una conceptualización específica. Ya iniciada esa década tiene lugar una primera elaboración

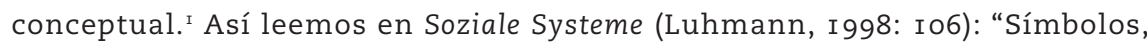
que - a diferencia de las denominaciones a algo distinto - son aquello mismo que designan".

Estas pocas palabras contienen el núcleo singular del concepto: designa la unidad de la remisión a sí mismo de algo que es capaz de remitirse a sí mismo, es decir, indica una identidad en el marco de un programa explícitamente dedicado a la diferencia. ${ }^{2}$ En trabajos posteriores, y hasta el fin de los días del autor, este núcleo será conservado. Así, a propósito de la distinción entre signo y símbolo, agregará un tiempo después:

Originariamente, el symbolon fue la representación o el testimonio de la unidad de dos piezas separadas que combinaban entre sí, sobre todo en los casos de adquisición de un determinado status a través de la hospitalidad. En estos términos, el symbolon representaba una unión mediante una separación. En el contexto semiótico esto puede tratarse únicamente a partir de la distinguibilidad (Unterschiedenheit) entre significado y significante, un hecho suficientemente comprehendido (designado) por el concepto de signo. Se puede hablar de símbolos en aquellos casos en que el signo designa la propia función de puesta en unidad (Vereinheitlichung) de lo separado. El símbolo sería, por tanto, la auto-designación (Selbstbezeichnung) del signo. Los signos simbólicos no son solo señales orientadoras que apuntan en dirección a otra cosa. Tampoco son meros portadores (Träger) de una referencia significante o de materializaciones del significante, sino que implican, además de todo eso, la indicación de la propia función, la indicación de la propia unidad recién formada del sentido del signo. De esta manera, los símbolos degradan la materialización de la puesta en signo hasta convertirla en un mero componente incapaz de actualizar por sí mismo el "sentido que tiene en realidad" el signo. Solo a través de la simbolización se hace posible distinguir el signo propiamente dicho del significante (Luhmann, I993: 67, trad. propia). ${ }^{3}$

Como se ve, a partir de aquella formulación el símbolo dejó de ser una palabra en la TGSS y se convirtió en un concepto. Con esta definición tendrá dos usos en los trabajos de Luhmann. Un primer uso, que denominamos uso ampliado porque está referido a la teoría del sentido, cuya función será indicar la unidad de las generalizaciones de sentido; y un segundo uso, que denominamos uso reducido porque está referido a los MCSG de la sociedad moderna, cuya función será indicar la unidad generalizada de las codificaciones binarias en referencia a la improbabilidad de la aceptación.

Ninguno de ambos usos ofrece una definición general del símbolo y de las simbolizaciones en relación con la comunicación. El uso ampliado está indeterminadamente referido al sentido. Por este motivo, primero, carece de referencias a la comunicación y es incapaz de establecerlas por sí mismo, ya 
que simplemente no se puede diferenciar sistemas psíquicos de sistemas sociales en el medio del sentido, porque allí participan por igual comunicaciones y consciencias, y, segundo, requiere una operación de reespecificación que conecte al símbolo con la operación comunicativa y lo situe así en el terreno de la teoría general de sistemas sociales.

El uso reducido está excluyentemente concentrado en la sociedad moderna funcionalmente diferenciada, por tanto, primero, no está disponible para sociedades no modernas o para sistemas parciales no funcionales, tampoco lo está para organizaciones, interacciones y/o sistemas de protesta, ${ }^{4} \mathrm{y}$, segundo, requiere una operación de generalización que permita situarlo en el terreno de la teoría general de sistemas sociales. En suma, a la conceptualización de símbolo y de simbólico realizada por Luhmann le falta una conexión general con la comunicación qua operación de sistemas sociales. Ello no quita, sin embargo, que sus contribuciones sean altamente significativas y ofrezcan elementos para operar la conexión faltante.

Consideramos así que, al estudiarse la conexión entre simbolizaciones y comunicación, se debe atender la formación y el funcionamiento comunicativos de los símbolos; si hay símbolos o procesos simbólicamente relevantes más allá (o más acá) de los MCSG y de la sociedad; si junto a los símbolos o simbolizaciones generalizados hay símbolos o simbolizaciones no generalizados o particularizados; e incluso, dentro del reducido ámbito de los MCSG, si hay simbolizaciones generalizadas cuya diferenciación (Ausdifferenzierung) no sea funcional. ${ }^{5}$ En vista de la heurística positiva (Lakatos, I974: 25-26) que abren estos interrogantes, entendemos que la tarea de definir en general la conexión del símbolo con la comunicación requiere precisar el concepto con el mayor grado de abstracción posible en relación con la comunicación, señalar las condiciones comunicativas de su formación y de su extinción, e indicar tanto sus referencias problemáticas como sus orientaciones funcionales.

Como se ve, la tarea excede largamente el marco de este trabajo. Nuestro objetivo aquí será examinar el uso restringido del símbolo en relación con los MCSG y ensayar la operación de generalización, ya que en este uso hay muchos más elementos relativos a la comunicación que en el primero, especialmente en lo relativo a la orientación a la aceptación, donde hay indicios de un intento de generalización ensayado por el propio autor. Con ello se aportará un primer esquema general que permita conectar símbolo y comunicación al nivel de la teoría general de sistemas sociales. Conservaremos sin embargo al primer uso en el horizonte de trabajo, no lo descartaremos, ya que nos servirá para controlar las propuestas.

Nuestra hipótesis sugiere que, en relación con la improbabilidad de la aceptación, la comunicación estiliza las expectativas frente a decepciones mediante la introducción de símbolos y simbolizaciones. A esta simbolización de 
la comunicación la denominaremos estilización simbólica de las expectativas de aceptabilidad y de rechazabilidad. Con ella designamos procesos pertenecientes al nivel de la teoría general de sistemas sociales aptos para ser reespecificados en los diversos planos de la diferenciación vertical (sociedades, organizaciones, interacciones, sistemas de protesta) y horizontal (sistemas parciales de la sociedad) de planos sistémicos.

Cabe una importante aclaración de la hipótesis, pues ella impacta de lleno en la teoría de los MCSG. De acuerdo con nuestra premisa, dicha teoría afronta dos opciones: o bien, (a) ella es generalizada e ingresa a la teoría general de sistemas sociales, volviéndose reespecificable en todos los niveles verticales y horizontales, ${ }^{6}$ o bien (b) permanece reducida a la teoría específica de la sociedad. Si se opta por (b), se afrontarían de todos modos dos sub-opciones, o bien (b.i) es ampliada al sistema de la sociedad en general y se indagan diferenciaciones de MCSG no-modernas y/o no funcionales, o bien (b.ii) permanece reducida a la teoría específica de la sociedad moderna y de la diferenciación funcional. Si bien Luhmann elaboró muchas de sus posiciones en dirección a b.ii, dejó indicios de las otras opciones y sub-opciones.

En cualquier caso, según nuestra hipótesis, ni a la sociedad moderna ni a los sistemas parciales ni a los MCSG se les podrá atribuir ya pretensiones de exclusividad sobre la comunicación simbólica. A propósito de esto, para saldar debidamente cuentas con el nuestro punto de partida, ensayaremos una sub-hipótesis, o hipótesis auxiliar, en la línea de la opción (a), según la cual, los MCSG constituyen una formación especial de comunicación simbólica asociada con códigos binarios y símbolos de mayor alcance, y donde la generalización es equivalente funcional de la normatividad. Así pues, no sólo los MCSG pueden orientarse a la improbabilidad de la aceptación en cualquiera de los planos verticales, sino que también pueden hacerlo hacia problemas de aceptabilidad tanto funcionales, como de estratificación, segmentación y centro-periferia, con independencia del primado que eventualmente adquirieran modalidades tanto en la forma primaria de la diferenciación de la sociedad y de los sistemas parciales de ella, como en las organizaciones, interacciones y protestas.

La exposición seguirá este orden: ensayaremos la generalización del concepto de símbolo de la teoría de los MCSG y luego la conectaremos con la teoría de las estructuras de expectativas, puntualmente con el concepto de estilización ante decepciones. Tras ello, saldaremos cuentas con la teoría de los MCSG y ampliaremos el concepto de comunicación simbólica resultante en los planos de la diferenciación vertical y horizontal de sistemas sociales. Finalmente, discutiremos los resultados alcanzados y extraeremos las conclusiones. 


\section{GENERALIZACIÓN DE LA FUNCIÓN DE LOS SÍMBOLOS EN RELACIÓN CON LA ACEPTACIÓN DE LA COMUNICACIÓN}

Cada vez que sistematizó la teoría de los MCSG, Luhmann destacó la importancia del concepto de "medio" (Luhmann, I974, I98I, I984, I997). Este designa el sustrato dónde el proceso de generalización simbólica es posible, así como su naturaleza. Sobre él descansa la arquitectura de la teoría y está encadenado con los otros pilares del fundamento operativo: los conceptos de sistema y de evolución. La sociología es entramada en las otras dos propiedades del concepto: lo "simbólico" y lo "generalizado". Mientras lo "generalizado" articula una dimensión de extensión o alcance - no técnica-propagativa - y una normativa, lo "simbólico" carga la dimensión comunicativa del concepto. Tomemos las palabras de Die Gesellschaft der Gesellschaft:

En el contexto del concepto "medios de comunicación simbólicamente generalizados", por "simbólico" se entiende - como en Parsons - que estos medios superan una diferencia y dotan a la comunicación con perspectivas de aceptación [...] Los medios simbólicamente generalizados transforman, de manera asombrosa, las probabilidades del no en probabilidades del sí; por ejemplo, al hacer posible ofrecer pagos por bienes o servicios que se desean obtener. Son 'simbólicos' en cuanto utilizan la comunicación para producir el acuerdo que de por si es improbable. Pero son, al mismo tiempo, diabólicos en cuanto que al realizar este cometido producen nuevas diferencias. Así, un problema de comunicación especifico se resuelve mediante un nuevo arreglo de unidad y diferencia: quien puede pagar obtiene lo que desea; quien no, no... (Luhmann, 2007: 248, primer y último énfasis nuestros).

Como se ve, la función primordial de probabilizar la aceptación recae exclusivamente en lo simbólico. A dicha propiedad remitimos entonces los interrogantes generales ¿Qué es, comunicativamente hablando, un símbolo, y qué lo caracteriza como tal? ¿Cómo se forma socialmente y cómo logra tratar exitosamente la improbabilidad de la aceptación? Volvamos a las palabras del autor:

El concepto de "símbolo, simbólico" se utiliza - sobre todo desde el siglo XIX - en un sentido muy general y difuso; muchas veces casi como sinónimo de "signo" (Zeichen). Pero con eso se haría a sí mismo superfluo. Para reponerle su sentido preciso lo limitamos a aquel caso en que el signo designa a su vez su propia función, cuando se vuelve reflexivo. Por 'propia función' entendemos: representación (Darstellung) de la unidad de significante y significado (Bezeichnendem und Bezeichnetem). Mediante la simbolización se expresa (y por eso se hace comunicable) que en la diferencia reposa una unidad y que lo separado está unido, de tal suerte que se puede utilizar el significante en representación de lo significado - y no tan solo como alusión a lo significado (Luhmann, 2007: 247-248, énfasis nuestro)

Esta definición establece, en primer lugar, que hay signos y que estos pueden volverse reflexivos, en cuyo caso devienen símbolos. En segundo lugar, no hay signos inmanentemente simbólicos, sino que es imprescindible una operación de reflexión que los conforme como tales, y por esa misma razón ningún símbolo escapa a la posibilidad de volverse signo en la medida en que 
dicha reflexión, ora fracase (no se logra establecer unidad entre significante y significado), ora deje de utilizarse. En tercer lugar, en caso de formarse, el símbolo es funcional y produce una unidad significante denominada simbolización.7 Sobre este último punto no debemos, sin embargo, precipitarnos. La definición de símbolo es general, pero la descripción de su función no, sólo concierne a la sociedad. Por tanto, antes de pasar de una a la otra, cabe preguntarse ¿Qué sucede cuando la comunicación en general (re)introduce simbolizaciones? Dicho de una manera menos elíptica ¿Hay comunicación simbólica en general, es decir ajena a los MCSG?

En lo que sigue, las citas de Luhmann solo nos ofrecerán apoyaturas parciales, pues no fue un tema que el autor alemán profundizara. Examinemos otros pasajes:

Son medios autónomos en relación directa con el problema de la improbabilidad de la comunicación, aunque presuponen la codificación si/no del lenguaje y se hacen cargo de la función de hacer esperable la aceptación de una comunicación (die Annahme einer Kommunikation erwartbar zu machen) en aquellos casos donde el rechazo es lo probable. No surgen sino hasta que hay escritura - con la cual el rechazo de las pretensiones de sentido se hace todavía más probable. Estos medios responden al problema de que más información significa normalmente menos aceptación (Luhmann, 2007: 245).

De acuerdo con lo indicado, la función simbólica es "hacer esperable la aceptación", es decir, su función es elaborar aceptabilidad mediante simbolizaciones bajo condiciones lingüísticas de rechazabilidad. Los símbolos ni fuerzan ni imponen la aceptación, sino que modifican la distribución lingüística de expectativas y refuerzan la expectabilidad de aceptación. Luhmann sugiere asimismo una relación entre la simbolización y la información, ya que, si el incremento de información debilita la aceptabilidad, la reintroducción de símbolos contraría esa tendencia y refuerza la aceptabilidad bajo esa condición. ${ }^{8}$ A propósito de esto, se lee en la misma obra:

Aceptación y rechazo desencadenan recursiones diferentes. Esta reflexión explica que en uno de los caminos - propiciado por los rechazos - surgen instituciones de superación de los conflictos que deben focalizarse sobre casos particulares imprevisibles de divergencia de opiniones y antagonismo; mientras en el otro camino surge una semántica positiva del sentido aceptado, que - por decirlo así madura en el proceso de la reutilización, de la condensación, de la abstracción [...] Los medios simbólicamente generalizados son uno de los resultados de este proceso. (Luhmann, 2007: 246, énfasis nuestro)

En relación a reforzar la aceptabilidad, o a hacerla menos improbable, en condiciones informacionales que la debilitan, recogemos del pasaje anterior que los elementos asociados a la función simbólica son la reutilización, la condensación y la abstracción, cuyo contexto de emergencia es la semántica. En este sentido, en la medida en que la reflexión del signo es operada en la comunicación, la formación y recursión (i.e. la dinámica) simbólica son actua- 
lizadas cuando son actualizadas, carecen de existencia exterior y suponen sistemas y procesos autorreferenciales. Así pues, la orientación funcional de la simbolización es formar reflexivamente una unidad significante en cuyo horizonte interno la reutilización, la condensación y la abstracción hacen esperable la aceptación de la comunicación.

Esta orientación funcional desemboca en la especial vinculación entre selección y motivación de la comunicación simbólica. Al respecto indica el autor:

El problema combinatorio es resuelto a través de la solución de la relación circular entre selección y motivación (una condiciona a la otra), es decir a consecuencia de que el condicionamiento de la selección es convertido en factor de motivación. Se puede aceptar una comunicación sobrecargada de exigencias (zugemutete Kommunikation) si se sabe que su elección obedece a determinadas condiciones; $\mathrm{y}$, al mismo tiempo, quien comunica tales exigencias desmesuradas puede - siguiendo estas condiciones - acrecentar la probabilidad de aceptación y con ello alentarse a sí mismo a la comunicación. De esta manera se resuelve - o al menos se normaliza - el doble problema de la decepción (Täuschung) y de la aceptación (Akzeptanz). La seguridad de que tales condiciones se tomen en cuenta aumenta, aunque ellas son por su parte altamente selectivas y de ningún modo abarcan la constelación deseada por completo; se señala esta autodeterminación (Selbstfestlegung) mediante el empleo de los símbolos correspondientes, los que atestiguan el uso del médium, de manera tal que se gana la perspectiva de la aceptación de la comunicación (Luhmann, 2007: 249, énfasis nuestro, trad. levemente modificada).

La relación circular entre selección y motivación surge cuando se logra motivar selectivamente y seleccionar motivadamente. Es indispensable para ello que la comunicación designe inequívocamente la incorporación de la expectativa de selección entre las condiciones de la motivación. La simbolización es la encargada de tal designación y de distribuir, gracias a ello, las expectativas de aceptabilidad reforzadas con este peculiar factor de motivación selectivo. En la cita, la idea es derivada del concepto de médium de los MCSG, pero en la medida en que concierne a la constelación Alter ego/alter Ego, cabe generalizarla.

Nos quedamos con el principio de que la simbolización altera y redistribuye las expectativas de aceptabilidad simbolizadas mediante su unidad significante, y coloca la aceptación bajo un horizonte interno de expectabilidad circular entre selecciones y motivaciones simbólicamente orientadas a la reutilización, la condensación y la abstracción de los signos reflexivamente dispuestos a tales efectos. Así pues, tanto la selección como la motivación se vuelven esperables en tanto simbolizadas. Esto se debe a que el símbolo dona unidad significante al reforzamiento selectivo-motivacional de la aceptabilidad. Es decir, las expectativas simbolizadas, orientadas a reforzar la aceptabilidad, forman un doble círculo entre la circularidad significante (unidad del símbolo) y la circularidad selectiva (motivaciones selectivas, selecciones motivadas). El 
lugar central que ocupa la expectabilidad de aceptación nos sugiere continuar el desarrollo en el terreno de la teoría de las expectativas comunicativas de la TGSS.

\section{ESTILIZACIÓN DE LAS EXPECTATIVAS DE ACEPTACIÓN}

Hasta aquí indicamos que la simbolización, cuando es introducida en referencia a la improbabilidad de la aceptación, se orienta, por un lado, a desplazar el umbral de rechazabilidad en las expectativas de empalme y, por otro lado, a convertir las posibilidades problemáticas de la aceptabilidad en posibilidades abiertas. Para esto último son reforzados simbólicamente determinados signos, condiciones y expectativas en el horizonte de la selección. En estos términos, proponemos considerar que la simbolización establece una relación con la decepción de expectativas y con la explotación de la incertidumbre y la inseguridad. ${ }^{9}$

Acerca de la decepción, el aseguramiento y la estilización de expectativas en general expresó Luhmann (I998: 268) lo siguiente:

La formación de expectativas iguala un gran número de acontecimientos altamente heterogéneos al denominador común de la decepción de la expectativa, y designa asílíneas de tratamiento. Uno se ve prácticamente obligado a reaccionar ante la decepción. Esto se puede hacer adaptando la expectativa a la situación de la decepción (aprendizaje) o exactamente al revés, sosteniendo la expectativa a pesar de la decepción e insistiendo en una conducta de expectativa. Se puede preestructurar, en el interior del sistema, cuál modo de reacción se escoge, y sólo de allí depende hasta dónde y en qué dirección uno debe ocuparse de los orígenes de la discrepancia.

El planteo de Luhmann, que sigue aquí muy estrechamente el planteo original de Johan Galtung (I959: 2 I6ss), ${ }^{\text {Io }}$ procura encauzar el tratamiento de reacciones y anticipaciones ante decepciones a través del concepto de estilización de expectativas. Se habla entonces de decepción de expectativas cuando un elemento no las satisface y se abre un horizonte de reelaboración orientado a reabosorber inseguridades en nuevas expectativas, siendo así estilizadas frente a la posibilidad de decepción.

Ensayaremos a continuación una caracterización de la simbolización como estilización de las estructuras de expectativas relativas a la decepción. A tal efecto debemos primeramente contradecir a Luhmann (2007: 245, trad. levemente modificada), quien indicó que "Los medios de comunicación simbólicamente generalizados (sobre todo el derecho) no sirven primariamente para asegurar (Absicherung) las expectativas contra las decepciones (Enttäuschungen)". En rigor, desde la perspectiva de la TGSS, nada sirve a tal efecto, ya que simplemente no es posible alcanzar semejante protección o aseguramiento, más bien se trata de identificar y, eventualmente, explotar la inseguridad, volviendo, por ejemplo, segura o relativamente cierta la aparición de decepciones. Y si bien es cierto que ni las generalizaciones simbólicas en particular 
ni las simbolizaciones en general forman expectativas normativas o cognitivas respecto de las decepciones, no es menos cierto que el esquema de Galtung y de Luhmann es cuestionable.

Lindemann (2009: I94Ss) señala al respecto que el planteo de Luhmann, al igual que el de Galtung, ofrece poca plasticidad para el análisis teórico y empírico de la relación entre decepción y estilización, particularmente en lo concerniente a las expectativas cognitivas. ${ }^{\text {II }}$ Suscribimos el cuestionamiento y agregamos que tampoco luce exhaustivo, ya que hay al menos una opción no descartamos que haya otras - de reabsorción de decepciones no contemplada en él, a saber, la opción reflexiva: estilizar expectativas para decepcionar a la decepción. En ese punto, no sólo la inseguridad esperable es estilizada en seguridad esperable, sino que además las expectativas relativas a la aceptabilidad ganan precisión dentro del horizonte de expectativas. Es decir, la simbolización hace esperable que la decepción sea rechazada a nivel operativo y sea decepcionante a nivel estructural.

Lo dicho, la simbolización establece una relación con la decepción de expectativas. Aclaramos ahora que tal relación es negativa, pues consiste en difuminar la decepcionabilidad. Esta difuminación se realiza mediante una elaboración mucho más precisa en materia significante de la aceptabilidad en el horizonte de expectativas. De esta manera, se observa que el doble círculo de las simbolizaciones explota la incertidumbre propia de las expectativas inseguras y redistribuye tales expectativas a los efectos de disparar procesos de estilización dirigidos específicamente a las decepciones por un lado, y de fijar y precisar mediante dicha estilización el predominio de un tratamiento reflexivo de las expectativas por otro lado. En otras palabras, la introducción de simbolizaciones en la regulación de la improbabilidad de la aceptación está orientada a decepcionar la decepción. Para ello forma mediante símbolos un umbral de decepcionabilidad para las decepciones.

Entendemos entonces que, en relación con la improbabilidad de la aceptación, la introducción de símbolos y de simbolizaciones dispara procesos que transforman las inseguridades esperables dadas y la reabsorción anticipada de decepciones establecida. En este sentido, la simbolización orientada a decepcionar las decepciones participa de la reelaboración de expectativas y de su estilización. La unidad significante del símbolo fija y precisa de manera novedosa las referencias de las posibilidades de aceptación. Gracias a la alteración y la estabilización repentina del horizonte de expectativas que logran las simbolizaciones, las decepciones esperadas enfrentan un hasta entonces inexistente umbral simbólico de inseguridad; sus posibilidades abiertas se convierten en posibilidades problemáticas, ya que se vuelven imprecisas y sus empalmes se difuminan. Al mismo tiempo y de manera concomitante, los símbolos fijan y precisan otras referencias de empalme, haciendo que las expectativas para su aceptación muden sus posibilidades remotas o problemáticas 
en posibilidades abiertas. En consecuencia, entendemos que la estilización de expectativas que logra la simbolización consiste en redistribuir reflexivamente la expectatibilidad de la decepción en el horizonte de expectativas.

Como se ve, la simbolización no actúa positivamente en pos de la aceptación, sino que actúa negativamente en contra del rechazo. Simplifica la codificación no-lingüística de la aceptación y complejiza la codificación lingüística y no-lingüística del rechazo. De esa manera, facilita indirectamente la aceptación dificultando directamente el rechazo, y esto último es posible porque el rechazo ya no está orientado solo a la codificación del lenguaje, sino que también lo está hacia la codificación de los símbolos introducidos comunicativamente. En tal sentido, la diferencia específica de la decepción no es establecida en referencia a expectativas inseguras - como sugieren Luhmann y Galtung para las normas y las cogniciones -, sino que los símbolos hacen que la decepción remita a sí misma y ésta ve transformarse repentinamente su horizonte interno, volviéndose ella propiamente insegura, ya que la negación lingüística y el rechazo simbólico acaban diferenciándose como posibilidades de empalme negativo distintas: en el primer caso, la negación se difumina ante la precisión reflexiva del símbolo, mientras que, en el segundo caso, surge un umbral simbólico de contenido mínimo decepcionante para el rechazo.

Antes de dar el siguiente paso, recordemos que Luhmann, al igual que Habermas, no define la aceptación comunicativa de manera positiva, sino que lo hace mediante una doble negación: aceptar es rechazar el rechazo. Entonces, la diferenciación de posibilidades de decepción entre rechazo lingüístico y rechazo simbólico implica que, al introducir símbolos en las remisiones a expectativas orientadas a la decepción, la comunicación refuerza la decepción de la decepción y hace menos improbable el rechazo del rechazo (= la aceptación). De eso se trata, a nuestro entender, la estilización simbólica de las expectativas.

Los símbolos elaboran la decepción de manera decepcionante en el horizonte de expectativas, es decir estilizan la diferencia como símbolo decepcionante y estilizan su rechazo. Así, gracias a la estilización simbólica, las expectativas de decepción se vuelven inseguras, pues vuelven al rechazo decepcionante, y, por tanto, inseguro e inestable. Esto implica que la decepción de la decepción es estabilizada simbólicamente como rechazable en el plano de las expectativas, y que la distribución asimétrica de la rechazabilidad del símbolo decepcionante hace menos improbable el rechazo del rechazo en los empalmes y en las informaciones. De esta manera, la simbolización reelabora las expectativas hasta lograr que la decepción sea la diferencia, pero no a la inversa.

Antes de cerrar el apartado queremos volver un momento al umbral simbólico de contenido mínimo decepcionante. Lo dicho, la simbolización, a través de su estilización de la decepción, introduce en la comunicación una codificación secundaria del rechazo. De manera que, una vez introducidas las simbolizaciones, el rechazo y la decepción lingüísticos permanecen como posibilidades, 
pero se forma un umbral de expectativas de rechazabilidad nuevo que involucra al símbolo y establece que el rechazo de la simbolización es más efectivo si la involucra a esta negativamente. O incluso, en una dinámica más compleja, es más efectiva aún si introduce nuevas simbolizaciones que rechacen simbolizaciones precedentes. ${ }^{\mathrm{I} 2}$ Esto contiene un potencial espiralado, ya que se abre la posibilidad de contradicciones y de conflictos simbólicos en la comunicación.

Con esto último queremos dejar indicado que las simbolizaciones forman nuevas condiciones para la aceptabilidad, pero hacen lo propio también con las condiciones de la rechazabilidad, y que, por tanto, la indagación conjunta del proceso abre un auspicioso campo de trabajo tanto teórico como empírico para la TGSS.

\section{COMUNICACIÓN SIMBÓLICA EN GENERAL, MCSG EN PARTICULAR, Y DIFE- RENCIACIÓN VERTICAL Y HORIZONTAL}

En base a lo expuesto en el apartado anterior, cabe preguntarse ¿cuál es el impacto de un concepto general de comunicación simbólica en la teoría de los MCSG? Como es sabido, el tratamiento teórico general de los medios simbólicamente generalizados encuentra su origen en Parsons y tiene en Habermas y en Luhmann a sus continuadores más notables. Durante las últimas dos décadas su elaboración se ha consolidado y ofrece actualmente uno de los objetos de estudio más novedosos para la sociología de la modernidad, afirmándose incluso que su investigación constituye por derecho propio un programa (Chernilo, 2002; Mascareño, 2009).

Luhmann estableció una relación fuerte entre MCSG y sociedad moderna y privilegió el tratamiento de los primeros en conexión con esta última. Por lo dicho hasta aquí, entendemos que ese tratamiento teórico de los MCSG debe corregirse. Primero, es necesario establecer una relación general entre estos medios y la comunicación simbólica. Se requiere para eso, una generalización del concepto de medios de comunicación simbólicamente generalizados amén la cual queden situados en la teoría general de sistemas sociales; gracias a esto se alcanzará una definición estrictamente comunicativa de ellos, pudiéndoselos tratar como medios de comunicación antes que como medios de la sociedad. Segundo, es necesario establecer un conjunto de reespecificaciones conectadas con los distintos planos de la hoy día denominada diferenciación vertical de sistemas sociales. Con la formulación general en la mano, los MCSG son reespecificados en cada plano vertical: sociedades (incluida la moderna), organizaciones, interacciones, sistemas de protesta.

Hasta aquí se trata entonces de replicar, en una escala menor, el doble movimiento de generalización y reespecificación que aplicamos a la comunicación simbólica. Sin embargo, una vez vinculados los MCSG con el plano de las sociedades, hay que operar una segunda reespecificación, que los ponga en relación con la sociedad funcionalmente diferenciada. Ese movimiento nos devolverá al punto de partida de Luhmann. 
En cuanto al primer movimiento, la generalización del concepto de MCSG, entendemos que fue Luhmann el primero en esbozar su tratamiento en dos niveles, ora en la teoría general de sistemas sociales, ora en la teoría específica de la sociedad (moderna). Este esbozo quedó plasmado en un texto de I98I dedicado a las improbabilidades de la comunicación, lo que significa que el antecedente directo es previo al "giro comunicativo". Aquel planteo será luego integrado en el marco de referencia de la TGSS, ya que en Soziale Systeme (I 984), los MCSG quedan estrictamente referidos a la tercera improbabilidad de la comunicación, esto es, en referencia a la improbabilidad de que una comunicación sea aceptada como premisa de la comunicación subsiguiente. Según sus palabras:

Los desarrollos técnicos del lenguaje y de la propagación volvieron ciertamente más dudoso el éxito de la comunicación, es decir, cuál comunicación puede motivar la aceptación. Hasta bien entrada la modernidad se reaccionó ante esta improbabilización creciente con redoblados esfuerzos dedicados a las técnicas de persuasión, a la elocuencia como fin educativo, a la retórica como doctrina específica, a la exposición y discusión como arte del conflicto y de la imposición. La invención de la imprenta no logró que estos esfuerzos se volvieran obsoletos, sino que los reforzó aún más. El éxito no descansó sin embargo en tales tendencias conservadoras, sino en el desarrollo de medios de comunicación simbólicamente generalizados, los cuales están funcionalmente referidos a este problema.

Con simbólicamente generalizados queremos designar aquellos medios que emplean generalizaciones para simbolizar la relación entre selección y motivación, es decir, para representarla como unidad. Ejemplos importantes de esto son: la verdad, el amor, la propiedad/el dinero, el poder/el derecho, y hasta cierto punto también la fe religiosa, el arte y hoy día tal vez los "valores básicos" civilizatoriamente estandarizados. En todos estos casos, de muy diversas maneras y en referencia a muy diferentes constelaciones de interacción, se trata de condicionar de tal modo la selección de la comunicación que ésta accione como medio de motivación a la vez. Así la observancia de la propuesta selectiva puede quedar suficientemente asegurada. En la sociedad de nuestros días la más exitosa y extendida comunicación se desenvuelve sobre estos medios de comunicación y dirigen las oportunidades de formación de sistemas sociales hacia las funciones correspondientes. Una discusión ulterior de esto debe quedar en manos de la teoría de la sociedad (Gesellschaftstheorie). La teoría general de los sistemas sociales y de sus procesos comunicativos, sin embargo, puede servir para llamar la atención sobre el carácter altamente selectivo de estos modos de comunicación funcionalmente privilegiados (Luhmann, I998: I59, trad. levemente modificada).

Se ve aquí con claridad que el tratamiento de la improbabilidad de la aceptación pertenece a la teoría general de sistemas sociales, pues concierne a la comunicación qua unidad sintética de tres selecciones, y que el tratamiento de la formación de sistemas parciales funcionalmente orientados por los MCSG pertenece a la teoría de la sociedad. En este planteo de Luhmann está in nuce la generalización que queremos ofrecer, y cuya pregunta inicial es por tanto la siguiente ¿cuál es la especificidad de los MCSG? 
La respuesta tiene dos líneas de trabajo. Por un lado, resaltar la propiedad de medio generalizado, a la que el propio Luhmann se abocó, y para cuyo planteo no tiene nuestro desarrollo consecuencias mayores, y por otro lado, ligado estrechamente con nuestras afirmaciones, entendemos que lo propio de ellos es estabilizar la comunicación simbólica orientándola a problemas relativos a fórmulas de contingencia y estabilizando un código de dos valores, es decir un código binario en relación a un símbolo (verdad, poder, dinero, amor). La disposición de dos valores para un símbolo genera una nueva distribución de las posibilidades comunicativas, pues éste tiene ahora un horizonte de aceptabilidad más preciso y complejo, pues cuenta con posibilidades de empalme y reflexivas del código. Así la decepción de la decepción, ya no tiene un valor simbólico, sino dos y coordina ahora dos reflexiones: la del signo y la del código.

En cuanto al segundo movimiento, la vinculación de los MCSG en relación con los planos de la diferenciación vertical, constituye una respuesta teórica a las consecuencias implicadas por la adquisición de una definición general de MCSG, a saber, ya no se trata sólo de una formación medial de la sociedad, sino de una formación de estructura general. Razón por la cual es necesario asociar aquella definición con un esquema que permita operar todas las reespecificaciones que el caso demande y donde la sociedad esté incluida.

El candidato natural dentro de la TGSS a tomar este lugar es la diferenciación vertical de planos de los sistemas sociales. Se trata de un esquema de larga data al que Luhmann elaboró y reelaboró con distintas denominaciones y conformaciones, y se lo considera el paso de la TGSS como programa teórico general a la TGSS como sociología aplicada. Típicamente la diferenciación vertical establece tres planos específicos e irreductibles entre sí de sistemas sociales: las sociedades, las organizaciones y las interacciones. De esto se sigue que tanto la improbabilidad de la aceptación como la reespecificación de la comunicación simbólicamente generalizada se dan en las interacciones, en las organizaciones, en las protestas y, por supuesto, en la sociedad. A las reespecificaciones en el plano de la sociedad y de la sociedad moderna nos dedicaremos en los movimientos tercero y cuarto. Pero ¿cómo tratar a los MCSG cuando no están necesariamente referidos ni a los sistemas parciales de la sociedad ni a la sociedad?

Hay que partir del pasaje ya citado donde Luhmann indica que los MCSG no suponen, ni única ni primordialmente, referencias a problemas funcionales de la sociedad, sino, en términos generales, la referencia a la improbabilidad de la aceptación imputada por Luhmann a toda comunicación como tal. Entonces, donde haya problemas de aceptación procesados de acuerdo al reforzamiento de la selectividad, por medio de la codificación binaria de la comunicación simbólica, estaremos ante problemas propios de MCSG. A este respecto, para mostrar la factibilidad y la pertinencia de nuestra propuesta e indicar algunos de los caminos que entrevemos para ella, nos apoyaremos en los indicios, hi- 
pótesis auxiliares y ejemplos que el mismo sociólogo alemán presentó para las organizaciones, para las interacciones, y también para la sociedad moderna, recién volveremos a intervenir con la reespecificación de la sociedad, y seguiremos la posición de Estrada Saavedra (2015) para las protestas.

En cuanto a las organizaciones formales, son definidas por Luhmann como sistemas sociales cuya cerradura operativa está basada en la comunicación de decisiones, están conformados por membresía y su estructuración se orienta a la búsqueda de objetivos. La comunicación de decisiones explicita su contenido (la dirección adoptada) a la luz de las posibilidades descartadas (las direcciones factibles que se decidió no tomar). Son consideradas adquisiciones evolutivas de la modernidad, ya que suponen determinados rendimientos del sistema jurídico (personerías, contratos), del sistema económico (mercado laboral, salarios, pago dinerario) y del sistema educativo (certificaciones), que recién se conforman con el primado de la diferenciación funcional (Luhmann, 20Io: 438). Luhmann observó la especificidad y complejidad de la relación de las organizaciones con los MCSG. A propósito de ella introdujo una hipótesis notable, pero aún poco considerada, a saber: la diferenciación funcional de los MCSG genera interdependencias entre estos medios de consecución y las organizaciones profundizan la diferenciación funcional mediante la fractura de tales interdependencias (Interdependenzunterbrechungen) (Luhmann, 2010: 452ss). Esto se debe a que las organizaciones son sistemas autopoiéticos, no estructuras de la sociedad, y por lo tanto tienen sus maneras específicas de afrontar la improbabilidad de la aceptación. Esto significa que las organizaciones procesan la comunicación simbólicamente generalizada mediante decisiones, y observan los contextos comunicativos de los sistemas parciales funcionalmente diferenciados como un entorno interno distribuido en términos de explotación de la incertidumbre.

En cuanto a las interacciones, son sistemas sociales basados en la copresencia simultanea de los participantes. Están especialmente orientados por la distinción temas/aportes y su auto-organización es particularmente lábil, careciendo sus estructuras de expectativas de la capacidad de transformarse sin transformar al sistema como tal. Si bien Luhmann no se dedicó expresamente a su relación con los MCSG, en diversas ocasiones identificó interacciones específicamente relacionadas con tales medios de consecución, a saber, las transacciones donde bienes o servicios son ofrecidos y adquiridos y donde un pago es realizado, las reuniones políticas de tipo mitin donde se discuten cursos de acción posible, las discusiones dentro de un congreso científico, o las declaraciones de parte o incluso los careos dentro de un proceso jurídico, o las clases dentro de una institución educativa (Luhmann, I986: 97ss).

En cuanto a las protestas, seguimos a Estrada Saavedra (20I5) quien las caracterizó mejor que el autor alemán, al establecer que estos sistemas por su constitución y reproducción mediante comunicaciones están orientadas al 
conflicto. Tales comunicaciones se expresan temáticamente como movilizaciones de protesta en contra de diferentes oponentes (como el gobierno, las organizaciones eclesiales, las empresas, los medios de comunicación) o de las consecuencias no previstas de las organizaciones de los sistemas funcionales de la sociedad (como la política, el derecho, la economía, la ciencia o el arte). Esto ancla las referencias problemáticas en las estructuras del mundo de la vida, y no en una "colonización" de él por los sistemas funcionales, lo cual modifica el enfoque sobre la relación entre protesta y MCSG, ya que los sistemas de protesta convierten problemas reconocidos en demandas contestatarias. De esta manera, se inicia una comunicación de conflicto que cuestiona las operaciones de organizaciones, de los sistemas funcionales o, en su caso, las exclusiones que generan las distinciones trazadas por estos últimos que ponen en peligro o subordinan al sistema de protesta. Esto estabiliza la relación entre sistemas de protesta y conflicto social. Es una relación selectiva con elementos funcionales específicos: constelación estabilizada de oponentes, estrategias y disputas de recursos, catalización de problemas en demandas y semánticas propias centradas en narraciones contestatarias.

Dicho lo cual ingresamos al tercer movimiento, la reespecificación de los MCSG en el plano vertical de las sociedades. La sociedad es definida como un sistema social omniabarcador, en cuyo nivel operativo no tiene un sistema social que la abarque. Se trata de un sistema cerrado operativamente, autopoiético y autorreferencial, capaz de autoobservación. Su operación específica es la comunicación y su función es la regulación de la diferenciación sistémica entre comunicación y no-comunicación.

Desde los albores de su programa, Luhmann procuró distanciarse de Parsons en relación a los medios simbólicamente generalizados. Su principal operación al respecto fue alejarse de esquemas de tipo explanans-explanandum entre sociedad y MCSG, evitó así deducir a los MCSG a partir de la sociedad, y optó por explorar la diferenciación de ellos en relación a problemas, luego improbabilidades, de aceptación específicos. Subrayamos esto: la orientación es hacia problemas de aceptación, por tanto, más allá de que Luhmann se concentró en los problemas funcionales de la modernidad, nada impide que haya formación de MCSG en relación a otro tipo de problemas de referencia en otras sociedades. De hecho, es el mismo autor quien, primero, sugiere repetidamente que la magia, la religión y la moral son medios de consecución simbólicamente generalizados (Luhmann, 2007: I77ss, 3IOss, 402), y, segundo, identifica en sus análisis de la relación entre semántica y estructuras societales, simbolizaciones generalizadas relativas a la dominación señorial durante el Sattelzeit (Luhmann, I980: 29, 32, 75ss). En estos ejemplos del propio Luhmann cabe indicar la existencia de consecución simbólicamente generalizada no funcionalmente diferenciada, cuya referencia societal es más amplia que la sociedad moderna. 
Esto permite explorar medios de consecución con referencias problemáticas tanto funcionales como no funcionales. Así se evita una asociación excluyente entre modernidad y MCSG, pero sobre todo se abre el panorama de investigación de MCSG para MCSG no funcionalmente diferenciados, sino estratificados, segmentarios o centro/periféricos, u otras variantes propias de ellos. Y esta indagación puede darse en todas las sociedades, sea en la moderna, sea en formaciones ya extintas. Así vistos, no sólo la magia, la religión y la moral serían casos, sino también otras formas de diferenciación de medios como la estratificación (los juramentos feudo-vasalláticos, la posesión de caballo o de determinado armamento entre caballeros, la manualidad del trabajo), la segmentación (los matrimonios, las agremiaciones por oficio), la centro/ periferia (las luchas por la continuidad de la esclavitud en tierras haitianas libradas por los revolucionarios franceses, la conformación de virreinatos como regímenes de gobierno y administración en las posesiones coloniales de la corona castellana). La investigación regional, incluidas aquellas consagradas a América Latina, ya no encontrarán en los MCSG un criterio de oscilación entre lo moderno y lo no moderno, ya que ahora se pueden abordar problemas funcionales, pero también problemas de estratificación, segmentación, etc... Y esto puede ser replicado, además, en la misma sociedad funcionalmente diferenciada junto a las estructuras de los MCSG funcionalmente diferenciados. Incluso en la diferenciación interna de los sistemas parciales funcionales globales, se observa MCSG orientados a la estratificación (la subordinación en esquemas jerárquicos) o a la segmentación (la diplomacia en las relaciones entre Estados), particularmente en los sucesivos procesos de diferenciación interna de ellos.

En síntesis, la reespecificación de los MCSG con la sociedad debe evitar tanto la deducción como la reducción modernista, para así observar la simbolización generalizada y la codificación de la aceptación ante problemas estratificados, segmentarios, centro/periféricos y/o funcionales, entre otros. En este movimiento debe contemplarse seriamente la posibilidad de que la teoría de la sociedad de la TGSS se convierta en una teoría de sistemas y de medios.

En cuanto al cuarto y último movimiento, la segunda reespecificación de los MCSG dirigida a conectarlos con la sociedad moderna, representa la teoría canónica de Luhmann respecto de los MCSG. Desde muy temprano en la evolución de su programa de investigación, Luhmann estableció que la principal referencia sistémica de los MCSG es el sistema de la sociedad, más puntualmente el de la sociedad mundial funcionalmente diferenciada. Solo indicaremos que la teoría sistémica de la sociedad considera a los MCSG formaciones de estructura comunicativa específicamente orientados a la consecución frente a problemas funcionales de referencia. Sus características distintivas son: 
integrar motivación y selección en un mismo esquema, de manera tal que frente al problema de referencia y su consecución la selección misma sea la motivación,

conformar y estabilizar un código binario con valores asimétricos, pero con igual valor de empalme, gracias al cual las comunicaciones referidas al problema son distribuidas y codificadas de manera que la velocidad y el tempo de la distribución favorezca la consecución.

estabilizar la consecución en la variación, es decir reestabilizar de forma permanente empalmes novedosos ante decepciones frecuentes

catalizar la formación de sistemas funcionalmente diferenciados

estabilizar el código binario como esquema de observación de segundo orden, tanto del sistema como del entorno del sistema, integrando las relaciones intersistémicas.

En virtud de la asociación fuerte entre las características de los MCSG y las características de la diferenciación funcional de la forma primaria de diferenciación de la sociedad, aquéllos son consagrados a estructuras fundamentales de ésta. Su relación, tanto con los sistemas parciales funcionalmente diferenciados como con los procesos estructurales de diferenciación funcional de la sociedad, la colocan en un sitio privilegiado dentro de la teoría luhmanniana de la sociedad. Su ejemplificación fue abundantemente ofrecida por el propio Luhmann, tanto en las monografías sobre sistemas parciales como en los trabajos sobre el sistema de la sociedad, pero también en los estudios, inscriptos en la sociología del conocimiento sobre la relación entre estructura de la sociedad y semántica. ${ }^{13}$

\section{CONCLUSIONES}

A lo largo de este trabajo delineamos una versión parcial de un concepto general de comunicación simbólica desde las premisas revisadas de la TGSS de Niklas Luhmann. Nuestro eje de trabajo fue la relación entre símbolo y simbolizaciones y la improbabilidad de la aceptación, dejando abierta la posibilidad para otras elaboraciones en dirección a la improbabilidad del entendimiento. Para su elaboración operamos una generalización de los elementos signo/ símbolo, la aceptabilidad, los atributos de reutilización, condensación y abstracción, y la vinculación circular entre selección y motivación del concepto de MCSG del sociólogo alemán.

Luego proyectamos el esquema resultante de la generalización en el terreno de la teoría de las expectativas qua estructura de sistemas sociales del autor. En ese ámbito establecimos una conexión entre anticipación de inseguridad, decepción reflexiva y aceptabilidad, que nos condujo a un concepto de simbolización que designa un tipo de estilización de las estructuras en relación a la decepción, que se caracteriza por precisar las expectativas de aceptabilidad 
y difuminar las de rechazabilidad, aumentando mediante las codificaciones adicionales del símbolo la probabilidad del rechazo del rechazo (= aceptación) en el empalme de operaciones. Tras ello, a fin de saldar cuentas con nuestro punto de partida, proyectamos esta elaboración conceptual en la teoría de MCSG y logramos una considerable ampliación de ella: generalizamos también el concepto de MCSG, al que entendimos como una formación especial de comunicación simbólica asociada con códigos binarios y símbolos de mayor alcance, y planteamos un conjunto significativo y heurísticamente positivo de reespecificaciones tanto verticales como horizontales.

Los distintos resultados alcanzados se han mostrado congruentes con las hipótesis iniciales principal y auxiliar. La evaluación final nos indica que la versión preliminar del concepto de comunicación simbólica que alcanzamos se muestra útil para introducir nuevas distinciones, tanto en la investigación sistémica de la comunicación en general como de los MCSG en particular, siendo apto entonces para usos empíricos o aplicados y para desarrollos conceptuales y teóricos ulteriores referidos al problema estudiado.

Recibido I6/5/20I9 | Revisado 20/8/20I9 | Aprobado 28/8/20I9

Sergio Pignuoli Ocampo es doctor en ciencias sociales de la Universidad de Buenos Aires. Actualmente se desempeña como investigador de carrera del Consejo Nacional de Investigaciones Científicas y Técnicas con sede en el Instituto de Investigaciones Gino Germani y como docente regular de la cátedra "Niklas Luhmann y la sociología de la modernidad". Desarrolla investigación en teoría sociológica, teoría y epistemología de sistemas sociales y teoría de la comunicación y es investigador responsable del PICT 2015-007I: "La tensión entre diadismo y dualismo en las teorías sociológicas contemporáneas. Un estudio sistemático de los programas de Luhmann, Latour, Habermas, Schütz, Garfinkel, Giddens y Archer", financiado por BID y ANPCyT. 


\section{NOTAS}

I De acuerdo con nuestro relevamiento, no es necesario reconstruir la evolución de las apariciones del término símbolo (Symbol) en la obra de Luhmann, pues, previos a Soziale Systeme, los usos del término son similares al descripto en el cuerpo. Así lo muestran, por caso, los primeros tres volúmenes de Soziologische Aufklärung [Ilustración sociológica] y los primeros dos de Gesellschaftsstruktur und Semantik [Estructura de la sociedad y semántica]. Quizá las investigaciones del Luhmann-Archiv ofrezcan en un futuro materiales para enriquecer su análisis diacrónico. Agradezco a uno de los árbitros anónimos la posibilidad de hacer esta aclaración.

2 Algo similar ocurre con el concepto tardío de Eigenwert [auto-valor] (Luhmann, I997: 394ss), aunque aquí optamos por un abordaje de tipo comunicativo y por tanto no lo ensayamos, es posible desarrollar el concepto sistémico de símbolo como Eigenwert.

3 Debo esta referencia al Prof. Antonio Brasil Jr. A propósito del pasaje, cabe agregar que Luhmann empalmó su concepto de símbolo con la tesis de Novalis sobre la conexión entre simbolización y mistificación.

4 Seguimos el concepto de sistema de protesta de Estrada Saavedra (20I5), quien revisa y reformula el concepto de movimiento de protesta de Luhmann (I996a).

5 Luhmann empleó el concepto Ausdifferenzierung [literalmente, diferenciación hacia afuera] mayormente en relación a sistemas, pero también lo hizo en relación a los MCSG y sus códigos (por caso Luhmann, I974: 240-I). Esto permite indagar en qué medida ambas formaciones, sistemas y medios, se diferencian con principios propios, y cómo ambas diferenciaciones pueden converger (hipótesis "catalítica" entre medios y sistemas), pero también pueden divergir.

6 En este aspecto sería conveniente revisar las confrontaciones sistemáticas entre las teorías de medios de Luhmann y Parsons, ya que la propuesta de este último contemplaba ya una vasta generalización de los medios y los símbolos en el plano general de la acción, y es factible pensar que el papel de concepto de intercambio sea más apropiado que el de comunicación para esa avanzada 
orientación general. Debo esta sugerente idea a uno de los árbitros anónimos que juzgó el artículo.

7 Ciertamente, este pasaje echa luz a la distinción entre simbólico y diabólico del párrafo anterior. Los MCSG introducen simbolizaciones mediante las cuales logran reorientar, gracias a la unidad significante de estas, las improbabilidades de aceptación en problemas de codificación binaria, a través de la diferencia tecnificada de tales códigos.

8 Esto incorpora las simbolizaciones al análisis de autorreflexiones, autosimplificaciones y autodescripciones y semánticas, pues se asume que no es ajeno a la función de los símbolos modificar la distribución de expectativas, primero informacionales, luego semánticas, de un sistema social, siendo capaces de formar altas expectativas relativas al contenido (Zumutungsgehalt en los términos de Soziale Systeme (Luhmann, I984: 267ss), Sinnzumutungen en los de Die Gesellschaft (Luhmann, I997: 316)).

9 En Soziale Systeme Luhmann (I984: 440-44I; I998: 295) afirmó que el derecho y la ciencia son sistemas orientados a la decepción y, a propósito del primero, agregó que se trataba de una "disposición inmunitaria" (Immuneinrichtung). La misma posición se observa en La ciencia de la sociedad (Luhmann, I996b: I04-105) y en El derecho de la sociedad (Luhmann, 2005: I27Ss), aunque aquí ya se aprecia una oscilación. Hubiera sido enriquecedor que el autor justificara su cambio de mirada en 1997.

Io Debemos a Lindemann (2009: 194) la explicitación de la recepción dada por Luhmann de los trabajos de Galtung.

II A partir de este déficit, Lindemann (2009: 200ss) propuso una conexión con la "etno-teoría" [Ethno-Theorie], profundizó, diferenció y amplió el concepto de expectativa, logrando un catálogo y un esquema operacional más vasto.

I2 Esto ya está insinuado por Luhmann (I980: 79-80) en el concepto de "contrasimbolización" [Gegensymbolisierung], aunque se centra casi exclusivamente en la semántica.

I3 Catálogos de propiedades más amplios y exhaustivos se encuentran disponibles en Navas (I990), Chernilo (2002) y Mascareño (2009). Las características de los MCSG funcionales fueron objeto de interesantes críticas y ampliaciones. Por el lado de las ampliaciones, el propio Luhmann dejó abierto el número de MCSG, y así hicieron, por caso, Baec- 
ker (I994) que analizó en esos términos la asistencia social, Stichweh (20I6) hizo lo propio con el deporte de alto rendimiento y Lewandowski (20I2) con la pornografía. Por el lado de las críticas destacan los planteos de Künzler (1987), quién cuestionó las debilidades entrañadas en ella por la marginalización del lenguaje, y las objeciones de García Ruiz (I997) respecto del normativismo de base que Luhmann no pudo extirpar de los supuestos parsonsianos. Otra línea crítica pertenece a la TAC que cuestionó, desde una teoría alternativa de los MCSG, la homogeneización de los medios qua funcionalmente diferenciados (Habermas, I98I-II: 267ss).

\section{REFERENCIAS BIBLIOGRÁFICAS}

Baecker, Dirk. (1994). Soziale Hilfe als Funktionssystem der Gesellschaft. Zeitschrift für Soziologie, 23/2, p. 93-I Iо.

Chernilo Steiner, Daniel. (2002). La Teoría de los Medios Simbólicamente Generalizados como Programa Progresivo de Investigación. Revista Mad, 7.

Estrada Saavedra, Marco. (20I5). Sistemas de protesta. Esbozo de un modelo no accionalista para el estudio de los movimientos sociales. Tomo I. Ciudad de México: CES-COLMEX. Galtung, Johan. (1959). Expectations and interaction processes. Inquiry, 2/I-4, p. 213-234.

García Ruiz, Pablo. (I997). Los medios simbólicos ¿de comunicación o de intercambio? El legado parsoniano en Luhmann. Revista Anthropos, I73-I74, p. I00-II3.

Habermas, Jürgen. (I98I). Theorie des kommunikativen Handelns. 2 Bde. Frankfurt: Suhrkamp.

Künzler, Jan. (I987). Grundlagenprobleme der Theorie symbolisch generalisierter Kommunikationsmedien bei Niklas Luhmann. Zeitschrift für Soziologie, 16/5, p. 317-333. Lakatos, Imre. (1974). Historia de las ciencias y sus reconstrucciones racionales. Madrid: Tecnos.

Lewandowski, Sven. (2012). Die Pornographie der Gesellschaft. Bielefeld: Verlag.

Lindemann, Gesa. (2009). Das Soziale von seinen Grenzen her denken. Weilerswist: Velbrück. 
Luhmann, Niklas. (20I0). Organización y decisión. Ciudad de México: Herder.

Luhmann, Niklas. (2007). La sociedad de la sociedad. Ciudad de México: Herder.

Luhmann, Niklas. (2005). El derecho de la sociedad. Ciudad de México: Herder.

Luhmann, Niklas. (1998). Sistemas sociales. Barcelona/Ciudad de México/Bogotá: Anthropos/Iberoamericana/CEJA.

Luhmann, Niklas. (1997). Die Gesellschaft der Gesellschaft. 2. Bde. Frankfurt a.M.: Suhrkamp.

Luhmann, Niklas. (1996a). Protest: Systemtheorie und soziale Bewegungen. K-U. Hellmann (Hrsg.). Frankfurt a.M.: Suhrkamp.

Luhmann, Niklas. (I996b) La ciencia de la sociedad. Ciudad de México: Iberoamericana.

Luhmann, Niklas. (1993). Zeichen als Form. In: Baecker, D. (Hrg.). Probleme der Form. Frankfurt a.M.: Suhrkamp, p. 45-69. Luhmann, Niklas. (I986). Systeme verstehen Systeme. In: Luhmann, N. \& Schor, K. E. (Hrsg.). Zwischen Verstehen und Intransparez. Frankfurt a.M.: Suhrkamp, p. 72-II7.

Luhmann, Niklas. (1984). Soziale Systeme. Frankfurt a.M.: Suhrkamp.

Luhmann, Niklas. (I98I). The improbability of communication. International Social Science Journal, 33/I, p. I22-I3I.

Luhmann, Niklas. (1980). Gesellschaftsstruktur und Semantik. Studien zur Wissenssoziologie der modernen Gesellschaft. Bd. I. Frankfurt a.M.: Suhrkamp.

Luhmann, Niklas. (1974). Einführende Bemerkungen zu einer Theorie symbolisch generalisierter Kommunikationsmedien. Zeitschrift für Soziologie, 3/3, p. 236-255.

Mascareño, Aldo. (2009). Medios simbólicamente generalizados y el problema de la emergencia. Cinta Moebio, 36, p. I74-I97.

Navas, Alejandro. (I990). La teoría sociológica de Niklas Luhmann. Navarra: EUNSA/Ediciones Universidad de Navarra.

Stichweh, Rudolf. (20I6). Die soziologische Theorie der Inklusion und das Funktionssystem Sport. In: H. Meier, L. Riedl y M. Kukuk (Hrsg.). Migration, Inklusion und Integration. Bretten: Schneider, p. 37-47. 
Palavras-chave

Símbolo;

comunicación;

Teoría General de Sistemas

Sociales;

aceptación social;

medios de comunicación

simbólicamente

generalizados.

Keywords

Symbol;

communication;

General Theory of Social

Systems;

social acceptance;

symbolically generalized

media of communication.

\section{SÍMBOLO E COMUNICAÇÃO - RUMO A UM CONCEITO DE COMUNICAÇÃO SIMBÓLICA A PARTIR DA TEORIA GERAL DOS SISTEMAS SOCIAIS}

\section{Resumo}

Nesse artigo, examinamos a conexão geral entre símbolo e comunicação na Teoria Geral dos Sistemas Sociais de Niklas Luhmann e a problematizamos no sentido de elaborar um conceito geral de comunicação simbólica a partir dessa perspectiva. Analisam-se as possibilidades de tal elaboração, tendo-se optado por uma generalização do conceito de meios de comunicação simbolicamente generalizados (MCSG) devido à sua orientação para a improbabilidade de aceitação. Sugere-se a hipótese de que, em referência à improbabilidade da aceitação, a comunicação estiliza as expectativas de aceitabilidade, introduzindo símbolos e simbolizações. O esquema resultante é então projetado na teoria dos MCSG, e uma forte extensão da mesma é alcançada.

\section{SYMBOL AND COMMUNICATION - TOWARDS A CONCEPT OF SYMBOLIC COMMUNICATION BASED ON THE GENERAL THEORY OF SOCIAL SYSTEMS}

\section{Abstract}

In this paper we examine the general connection between symbol and communication in Niklas Luhmann's General Theory of Social Systems and problematize this connection in order to elaborate a general concept of symbolic communication based on this approach. This aim in mind, we analyse the possibilities afforded by such an elaboration, opting to extend the concept of symbolically generalized communication media (SGCM) through its orientation towards the improbability of acceptance. We advance the hypothesis that, in reference to the improbability of acceptance, communication stylizes the expectations of acceptability by introducing symbols and symbolizations. The resulting schema is then projected onto the SGCM theory, enabling a strong extension of the latter. 